\title{
СТАЦИОНАРЫ И ЦИКЛЫ РАЗНОСТНОГО УРАВНЕНИЯ С РАЗРЫВНОЙ ПРАВОЙ ЧАСТЬЮ
}

\author{
Р. 3. Абдуллин \\ Байкальский государственный университет, г. Иркутск, Российская Федерация
}

Информация о статье

Дата поступления

2 июня 2017 г.

Дата принятия к печати 20 ноября 2017 г.

Дата онлайн-размещения 27 ноября 2017 г.

\section{Ключевые слова}

Разностное уравнение; неподвижные точки; циклы; области притяжения

\begin{abstract}
Аннотация
В статье рассматривается заданное на отрезке скалярное нелинейное разностное уравнение с разрывной правой частью. При условии единственности и различном расположении относительно точки разрыва притягивающих неподвижных точек функций, описывающих правую часть слева и справа от точки разрыва, установлены области их притяжения. При отсутствии неподвижных точек, непрерывности и монотонности функций, описывающих правую часть, получены условия существования притягивающих циклов с периодом два и больше двух. Показано, что для выпуклых или вогнутых функций области притяжения этих циклов совпадают с отрезком, на котором определено уравнение.
\end{abstract}

\section{STEADY STATES AND CYCLES OF A DIFFERENCE EQUATION WITH DISCONTINUOUS RIGHTHAND SIDE}

\author{
Rafael Z. Abdullin \\ Baikal State University, Irkutsk, Russian Federation
}

\section{Article info}

Received

June 2, 2017

Accepted

November 20, 2017

Available online

November 27, 2017

\section{Keywords}

Difference equation; fixed points; cycles; domains

of attraction

\begin{abstract}
The article examines a scalar nonlinear difference equation with discontinuous right hand side on the interval. Under the condition of uniqueness and different arrangement with respect to the discontinuity of the attracting fixed points of functions describing the right-hand side to the left and to the right of the discontinuity, the domains of their attraction are established. In the absence of fixed points, continuity, and monotonicity of functions describing the right-hand side, conditions are obtained for the existence of attracting cycles with a period of two or more than two. It is shown that for convex or concave functions the domains of attraction of these cycles coincide with the interval on which the equation is defined.
\end{abstract}

Существование притягивающих неподвижных точек и циклов, существование циклов различных периодов, бифуркация циклов, наличие перемешивающих аттракторов, хаотическое поведение решений скалярного разностного уравнения $x(n+1)=\varphi(x(n))$ с непрерывной правой частью достаточно глубоко исследованы в работах А. Н. Шарковского и его учеников [1], в трудах А. П. Шапиро и С. П. Луппова [2], Ю. М. Свирежева и Д. О. Логофрета [3], М. В. Якобсона [4], T.-J. Li и J. A. Yorke [5] и других авторов. Области притяжения неподвижных точек и существование циклов скалярного разностного уравнения с разрывной правой частью изучены слабо [6].
В данной работе для скалярного разностного уравнения с функцией $\varphi(x)$, имеющей одну точку разрыва $x_{p}$ определяются области притяжения неподвижных точек функций, определяющих правую часть уравнения справа и слева от точки разрыва, условия существования циклов и их области притяжения для различных случаев расположения неподвижных точек относительно точки переключения.

Рассмотрим скалярное разностное уравнение с разрывной правой частью:

$$
x(n+1)= \begin{cases}f_{1}(x(n)), & x(n) \leq x_{p} ; \\ f_{2}(x(n)), & x(n)>x_{p}\end{cases}
$$


где $x \in[a, b] \subset R, n \in Z_{0}=\{0,1,2, \ldots\}, x_{p}=$ const, $a<x_{p}<b, f_{1}(x)$ и $f_{2}(x)$ - непрерывные на $[a, b]$ функции со значениями в $(a, b), f_{1}\left(x_{p}\right) \neq f_{2}\left(x_{p}\right)$.

Функции $f_{i}(x)$ имеют на $(a, b)$ по одной неподвижной точке $x_{i}^{*}, i=1,2$, которые удовлетворяют необходимым и достаточным условиям притяжения из работы А. Н. Шарковского «Разностные уравнения и их приложения» [1]:

$$
\forall x \in\left[a, x_{i}^{*}\right) f_{i}^{2}(x)>x, \forall x \in\left(x_{i}^{*}, b\right] f_{i}^{2}(x)<x .
$$

Здесь и далее $f_{i}^{k}-k$-я итерация фрункции $f_{i}$, т. е. $f_{i}^{0}(x)=x и f_{i}^{j}(x)=f_{i}\left(f_{i}^{j-1}(x)\right), f_{i}^{-1}$ - обратное к функции $f_{i}$ отображение. У А. Н. Шарковского показано, что из (2) следует

$$
\forall x \in\left[a, x_{i}^{*}\right) f_{i}(x)>x, \forall x \in\left(x_{i}^{*}, b\right] f_{i}(x)<x .
$$

Из доказательства достаточности условий (2) можно сорормулировать несколько предположений.

Лемма 1. Если $f_{i}[a, b] \subset(a, b)$ и выполнены условия (2), то областью притяжения стационарной точки $x_{i}^{*}$ разностного уравнения $x_{i}(n+1)=f_{i}\left(x_{i}(n)\right)$ является отрезок $[a, b]$.

Для разностного уравнения (1) определим области притяжения $P\left(x_{i}^{*}\right)$ неподвижных точек $x_{i}^{*}$ фрункций $f_{i}(x)$ при различном их расположении относительно точки переключения $x_{p^{\prime}}$ условия существования циклов некоторых периодов и области их притяжения.

Различное расположение неподвижных точек относительно точки разрыва с $x_{1}^{*}<x_{p}<x_{2}^{*}$.

Отрезок $\left[a, x_{p}\right]$ представим в виде $\left[a, x_{p}\right]=$ $=W \cup S$, где $W$ - множество точек, из которых решения уравнения (1) на некотором шаге $n$ попадают в $\left(x_{p}, b\right], S=\left[a, x_{p}\right] \backslash W-$ множество точек, из которых решения уравнения (1) не покидают $\left[a, x_{p}\right]$ при любых $n \geq 0$.

Промежуток $\left(x_{p}, b\right]$ представим в виде $\left(x_{p}, b\right]=V \cup G$, где $V$ - множество точек, из которых решения уравнения (1) на некотором шаге $n$ попадают в отрезок [a, $\left.x_{p}\right], G=\left(x_{p}, b\right] \backslash V-$ множество точек, из которых решения уравнения (1) не покидают $\left(x_{p}, b\right]$ при любых $n \geq 0$.

Пусть $W_{0}$ - множество точек перехода из $\left[a, x_{p}\right]$ в $\left(x_{p}, b\right]$ за один шаг, a $V_{0}$ - множество точек перехода из $\left(x_{p}, b\right]$ в $\left[a, x_{p}\right]$ за один шаг, т. е. $W_{0}=\left\{x \in\left[a, x_{p}\right]: f_{1}(x)>x_{p}\right\}$ и $V_{0}=$ $=\left\{x \in\left(x_{p}, b\right]: f_{2}(x) \leq x_{p}\right\}$. Тогда

$$
W=\bigcup_{k \geq 0} f_{1}^{-k} W_{0}, \quad V=\bigcup_{k \geq 0} f_{2}^{-k} V_{0}
$$

Прообразы $f_{1}^{-k} W_{0}$ и $f_{2}^{-k} V_{0}$ множеств $W_{0}$ и $V_{0}$, а также их подмножеств рассматривают- ся соответственно в промежутках $\left[a, x_{p}\right]$ и $\left(x_{p^{\prime}}\right.$ b]. Множества $S$ и $G$ являются положительно инвариантными для уравнения (1).

Решения уравнения (1), начинающиеся в $W$ при переходе в $\left(x_{p}, b\right]$, могут попасть в $G$ или $V$, а решения, начинающиеся в $V$, при переходе в $\left[a, x_{p}\right]$ могут попасть в $S$ или $W$. Поэтому множество $W$ разобьем на два непересекающихся подмножества $W_{1}^{V}$ и $W_{1}^{G}$ по переходам V и G:

$$
\begin{aligned}
& W_{1,0}^{V}=\left\{x \in W_{0}: f_{1}(x) \in V\right\} ; \quad W_{1}^{V}=\bigcup_{k \geq 0} f_{1}^{-k} W_{1,0}^{V} ; \\
& W_{1,0}^{G}=\left\{x \in W_{0}: f_{1}(x) \in G\right\} ; \quad W_{1}^{G}=\bigcup_{k \geq 0} f_{1}^{-k} W_{1,0}^{G} .
\end{aligned}
$$

Множество $V$ разобьем на два непересекающиеся подмножества $V_{1}^{W}$ и $V_{1}^{S}$ по переходам в $W$ и $S$ :

$$
\begin{aligned}
& V_{1,0}^{W}=\left\{x \in V_{0}: f_{2}(x) \in W\right\} ; V_{1}^{W}=\bigcup_{k \geq 0} f_{2}^{-k} V_{1,0}^{W} ; \\
& V_{1,0}^{S}=\left\{x \in V_{0}: f_{2}(x) \in S\right\} ; V_{1}^{S}=\bigcup_{k \geq 0} f_{2}^{-k} V_{1,0}^{S}
\end{aligned}
$$

Учитывая, что $V=V_{1}^{W} \cup V_{1}^{S}$ и $W=W_{1}^{V} \cup W_{1}^{G}$, множество $W_{1}^{V}$ разобьем на непересекающиеся подмножества $W_{2}^{V}$ и $W_{2}^{S}$ по переходам в $V_{1}^{W} \boldsymbol{V} V_{1}^{s}$, а множество $V_{1}^{W}$ - на подмножества $V_{2}^{W}$ и $V_{2}^{G}$ по переходам в $W_{1}^{V}$ и $W_{1}^{G}$ :

$$
\begin{aligned}
& W_{2,0}^{V}=\left\{x \in W_{1,0}^{V}: f_{1}(x) \in V_{1}^{W}\right\} ; \quad W_{2}^{V}=\bigcup_{k \geq 0} f_{1}^{-k} W_{2,0}^{V} ; \\
& W_{2,0}^{S}=\left\{x \in W_{1,0}^{V}: f_{1}(x) \in V_{1}^{S} ; \quad W_{2}^{S}=\bigcup_{k \geq 0} f_{1}^{-k} W_{2,0}^{S} ;\right. \\
& V_{2,0}^{W}=\left\{x \in V_{1,0}^{W}: f_{2}(x) \in W_{1}^{V}\right\} ; V_{2}^{W}=\bigcup_{k \geq 0} f_{2}^{-k} V_{2,0}^{W} ; \\
& V_{2,0}^{G}=\left\{x \in V_{1,0}^{W}: f_{2}(x) \in W_{1}^{G}\right\} ; V_{2}^{G}=\bigcup_{k \geq 0} f_{2}^{-k} V_{2,0}^{G}
\end{aligned}
$$

Аналогичным образом продолжим процесс разбиения множеств $W_{i}^{V}$ и $V_{i}^{W}$ для $i \geq 2$. Тогда $W_{i}^{V}=W_{i+1}^{V} \cup W_{i+1}^{(G, S)}$ и $V_{i}^{W}=V_{i+1}^{W} \cup V_{i+1}^{(G, S)}$, где для нечетных $i W_{i+1}^{(G, S)}=W_{i+1}^{S}$ и $V_{i+1}^{(G, S)}=V_{i+1}^{G}$, а для четных $i W_{i+1}^{(G, S)}=W_{i+1}^{G}$ и $V_{i+1}^{(G, S)}=V_{i+1}^{S}$. По построению разбиений множеств $W_{i}^{V}$ и $V_{i}^{W}$ решения уравнения (1), начинающиеся в $W_{i}^{(G, S)}$, при $i$ четном, попадают в $S$, а при $i$ нечетном - в $G$; решения из $V_{i}^{(G, S)}$ при $i$ четном попадают в $G$, а при $і$ нечетном - в $S$. Если при некотором $i W_{1}^{V}=\varnothing$ или $V_{1}^{W}=\varnothing$, то процесс разбиения завершается.

Пусть $\alpha_{0}$ - наибольшее на отрезке $\left[a, x_{p}\right]$ решение уравнения $f_{1}(x)=x_{p}$, не являющееся точкой экстремума функции $f_{1}(x)$, если такого решения нет, то принимаем $\alpha_{0}=$ a. Пусть $\beta_{0}$ - наименьшее решение уравнения $f_{2}(x)=x_{p}$ на $\left(x_{p}, b\right]$, если такого решения нет, то принимaем $\beta_{0}=b$. 
Лемма 2. Если для уравнения (1) с $x_{1}^{*}<x_{p}<x_{2}^{*}$ выполняются условия (2), то $\alpha_{0}<x_{1}^{*}, \beta_{0}>x_{p},\left[\alpha_{0}, x_{p}\right] \subset S,\left(x_{p}, \beta_{0}\right) \subset G$.

В силу леммы 1 и положительной инвариантности множеств $S$ и $G$ при $\alpha_{0}<x_{1}^{*}<$ $<x_{p}<x_{2}^{*}<\beta_{0}$ решения уравнения (1), начинающиеся в $S$ или попадающие в $S$, сходятся к $x_{1}^{*}$, а решения из $G$ или попадающие в $G$ сходятся к $x_{2}^{*}$, т. е. $S \subset P\left(x_{1}^{*}\right)$ и $G \subset P\left(x_{2}^{*}\right)$. Например, при $\alpha_{0}=$ а имеем $W=\emptyset, S=[a$, $\left.x_{p}\right], P\left(x_{1}^{*}\right)=\left[a_{1} x_{p}\right] \cup V, P\left(x_{2}^{*}\right)=G$; при $\beta_{0}=b$ получаем $V=\varnothing, G=\left(x_{p}, b\right], P\left(x_{1}^{*}\right)=S$, $P\left(x_{2}^{*}\right)=\left(x_{p}, b\right] \cup W ;$ из $\alpha_{0}=$ аи $\beta_{0}=b$ следует $W=\varnothing, V=\varnothing и P\left(x_{1}^{*}\right)=\left[a_{1} x_{p}\right], P\left(x_{2}^{*}\right)=\left(x_{p}, b\right]$.

Введем множества точек $\alpha_{i}$ и $\beta_{i}, i \geq 1$. При этом $\alpha_{i}-$ наибольшее на $\left[\mathrm{a}, \alpha_{i-1}\right]$ решение уравнения $f_{1}(x)=\beta_{i-1}$, если такого решения нет, примем $\alpha_{i}=a ; \beta_{i}-$ наименьшее на $\left[\beta_{i-1}, b\right]$ решение уравнения $f_{2}(x)=\alpha_{i-1}$, если такого решения нет, примем $\beta_{i}=b$. По построению $\alpha_{i} \geq a, \beta_{i} \leq \mathrm{b}$ и $x_{p}>x_{1}^{*}>\alpha_{0}>\alpha_{1}>\alpha_{2}>\ldots$, $x_{p}<x_{2}^{*}<\beta_{0}<\beta_{1}<\beta_{2}<\ldots$ Очевидно, что из конечности множества точек $\alpha$, следует конечность множества точек $\beta_{i}$ и наоборот.

Лемма 3. Пусть в уравнении (1) функции $f_{1}(x)$ и $f_{2}(x)$ имеют по одной неподвижной точке $x_{1}^{*}$ и $x_{2}^{*}, x_{1}^{*}<x_{p}<x_{2}^{*}$, для которых выполняются условия притяжения (2), $\alpha \neq a$ и на $\left[a_{1}\right.$ $\left.\alpha_{0}\right] f_{2} f_{1}(x)>x$ или $\beta_{0} \neq b$ и на $\left(\beta_{0}, b\right] f_{1} f_{2}(x)<x$. Тогда множества точек $\alpha_{i}$ и $\beta_{i}$ конечны.

Доказательство. Предположим противное - имеется бесконечное множество точек $\alpha_{i}$. По их определению $a<\alpha_{i+1}<\alpha_{i}<\alpha_{0}$, следовательно, последовательность $\{\alpha\}$ сходится и пусть $\alpha^{*}=\lim _{i \rightarrow \infty} \alpha_{i}$. Тогда из $f_{1}\left(\alpha_{i}\right)=\beta_{i-1}$ и $f_{2}\left(\beta_{i-1}\right)=$ $=\alpha_{i-2}$ следует $f_{2} f_{1}\left(\alpha_{i}\right)=\alpha_{i-2}$. Отсюда в силу непрерывности $f_{1}(x)$ и $f_{2}(x)$ получаем $f_{2} f_{1}\left(\alpha^{*}\right)=\alpha^{*}$, что противоречит условию $f_{2} f_{1}(x)>x$ на $\left[a, \alpha_{0}\right]$. Таким образом, при условиях леммы 3 множества точек $\alpha_{i}$ и $\beta_{i}$ конечны.

Очевидно, при конечности множеств точек $\alpha_{i}$ и $\beta_{i}$ наименьшее из $\alpha_{i}$ равно $a_{\text {, а наи- }}$ большее из $\beta_{i}$ равно $b$ и процесс разбиения множеств $W_{i}^{V}, V_{i}^{W}$ завершается за конечное число шагов.

Теорема 1. Пусть для уравнения (1) неподвижные точки $x_{1}^{*} и x_{2}^{*}$ удовлетворяют условиям притяжения (2) и $x_{1}^{*}<x_{p}<x_{2}^{*}$, на отрезке $\left[a, \alpha_{0}\right]$ выполняется неравенство $f_{2} f_{1}(x)>x$ при $\alpha_{0} \neq a$. Тогда, если выполняется неравенство

$$
\alpha_{k}<\min _{\left[x_{2}, b\right]} f_{2}(x) \leq \alpha_{k-1}
$$

то $V_{k+1,0}^{W}=\varnothing, W_{k+2}^{V}=\varnothing, V_{k}^{W}=V_{k+1}^{(G, S)}, W_{k+1}^{V}=W_{k+2}^{(G, S)}$, и при $k$ четном имеем

$$
\begin{gathered}
P\left(x_{1}^{*}\right)=S \cup V_{1}^{S} \cup W_{2}^{S} \cup \ldots \\
\ldots \cup V_{k-1}^{S} \cup W_{k}^{S} \cup V_{k}^{W} \cup W_{k+1}^{V}, \\
P\left(x_{2}^{*}\right)=[a, b] \backslash P\left(x_{1}^{*}\right),
\end{gathered}
$$

а при нечетном $k$ -

$$
\begin{gathered}
P\left(x_{1}^{*}\right)=S \cup V_{1}^{S} \cup W_{2}^{S} \cup \ldots \\
\ldots \cup V_{k}^{S} \cup W_{k+1}^{S} \quad P\left(x_{2}^{*}\right)=[a, b] \backslash P\left(x_{1}^{*}\right) .
\end{gathered}
$$

Если выполняется неравенство

$$
\beta_{k-1}<\max _{\left[a, x_{1}^{*}\right]} f_{1}(x) \leq \beta_{k},
$$

то $W_{k+1,0}^{V}=\varnothing, \quad V_{k+2}^{W}=\varnothing, \quad W_{k}^{V}=W_{k+1}^{(G, S)}$, $V_{k+1}^{W}=V_{k+2}^{(G, S)}$, и при $k$ четном имеем $P\left(x_{1}^{*}\right)=S \cup V_{1}^{S} \cup W_{2}^{S} \cup \ldots \cup V_{k-1}^{S} \cup W_{k}^{S} \cup$ $\cup V_{k+1}^{S}, \quad P\left(x_{2}^{*}\right)=[a, b] \backslash P\left(x_{1}^{*}\right)$, а при нечетном $k-P\left(x_{1}^{*}\right)=S \cup V_{1}^{S} \cup W_{2}^{S} \cup \ldots \cup W_{k-1}^{S} \cup$ $\cup V_{k}^{S} \cup W_{k}^{V} \cup V_{k+1}^{W}, \quad P\left(x_{2}^{*}\right)=[a, b] \backslash P\left(x_{1}^{*}\right)$.

Доказательство. Пусть выполняется неравенство (4). Тогда $\beta_{k+1}=b$ и $\beta_{k}<b$, отсюда и $f_{1}[a, b] \subset(a, b)$ следует $\alpha_{k+1}=a$. На $\left(x_{p^{\prime}} \beta_{k}\right)$ выполняется неравенство $f_{2}(x)>\alpha_{k-1}$, поэтому $\left(x_{p}, \beta_{k}\right)$ состоит только из подмножеств множеств $G, V_{1}^{(G, S)}, \ldots, V_{k}^{(G, S)}$. На промежутке $\left[\beta_{k^{\prime}}, b\right]$ в силу (3) и (4) выполняется неравенство $\alpha_{k}<f_{2}(x)<x$. Так как на $\left(\alpha_{k^{\prime}} x_{p}\right] f_{1}(x)<\beta_{k-1}$, то $\left(\alpha_{k^{\prime}}, x_{p}\right]$ состоит только из подмножеств множеств $S, W_{1}^{(G, S)}, \ldots, W_{k-1}^{(G, S)}, W_{k}^{(G, S)}$. Отсюда следует, что из $\left[\beta_{k^{\prime}}, b\right]$ возможны переходы только в эти подмножества. Поэтому $V_{k+1,0}^{W}=\varnothing n V_{k}^{W}=V_{k+1}^{(G, S)}$, а также $W_{k+2,0}^{V}=\varnothing n W_{k+1}^{V}=W_{k+2}^{(G, S)}$. Отсюда по построению множеств $W_{i}^{(G, S)}$ и $V_{i}^{(G, S)}$ следует, что области притяжения $P\left(x_{i}^{*}\right)$ при выполнении условия (4) для четного $k$ определяются как (5), а для нечетного $k$ - как (6). Для случая выполнения условия (7) доказательство проводится аналогичным образом. Если условие (4) или (7) выполняется при $k=0$, то, соответственно, принимается $\alpha_{k-1}=x_{p}$ или $\beta_{k-1}=x_{p}$.

При выполнении условий теоремы 1 наиболее простую структуру множества $S, G, W_{i}^{(G, S)}, W_{i}^{V}, V_{i}^{(G, S)}, V_{i}^{W}$, а значит, и области притяжения $P\left(x_{i}^{*}\right)$ имеют при условиях монотонности функций $f_{i}(x)$, а именно при $f_{1}(x)$, возрастающей на $\left[a, x_{1}^{*}\right]$, и $f_{2}(x)$, возрастающей на $\left[x_{2}^{*}, b\right]$, имеем $W=V=\emptyset n$ $P\left(x_{1}^{*}\right)=S=\left[a, x_{p}\right], P\left(x_{2}^{*}\right)=G=\left(x_{p}, b\right]$. Для $f_{1}(x)$, убывающей на $\left[a, \alpha_{0}\right]$ с $\alpha_{0} \neq a, n f_{2}(x)$, возрастающей на $\left[x_{2}^{*}, b\right]$, получаем $S=\left[\alpha_{0}, x_{p}\right], G=\left(x_{p}\right.$, b], $W=W_{0}=W_{1,0}^{G}=\left[a, \alpha_{0}\right)$ и $P\left(x_{1}^{*}\right)=\left[\alpha_{0}, x_{p}\right]$, $P\left(x_{2}^{*}\right)=\left[a, \alpha_{0}\right) \cup\left(x_{p}, b\right]$. При $f_{1}(x)$, возрастающей на $\left[a, x_{i}^{*}\right]$, и $f_{2}(x)$, убывающей на $\left[\beta_{0}\right.$, b] с $\beta_{0} \neq b$, имеем $S=\left[a, x_{p}\right], G=\left(x_{p}, b\right]$, 
$V=V_{0}=V_{1,0}^{s}=\left[\beta_{0}, b\right]$ и $P\left(x_{1}^{*}\right)=\left[a, x_{p}\right] \cup\left[\beta_{0}, b\right]$, $P\left(x_{2}^{*}\right)=\left(x_{p}, \beta_{0}\right)$. Если $f_{1}(x)$ и $f_{2}(x)$ убывают соответственно на $\left[a, \alpha_{0}\right]$ с $\alpha_{0} \neq a$ и $\left[\beta_{0}, b\right]$ с $\beta_{0} \neq b$, то $S=\left[\alpha_{0}, x_{p}\right], W=W_{0}=\left[a, \alpha_{0}\right), G=\left(x_{p}, \beta_{0}\right)$, $V=V_{0}=\left[\beta_{0}, b\right]:$

$$
\begin{gathered}
W_{i}^{(G, S)}=W_{i, 0}^{(G, S)}=\left\{\begin{array}{l}
{\left[\alpha_{i}, \alpha_{i-1}\right], \text { при четных } i,} \\
\left(\alpha_{i}, \alpha_{i-1}\right), \text { при нечетных } i,
\end{array}\right. \\
V_{i}^{(G, S)}=V_{i, 0}^{(G, S)}= \begin{cases}{\left[\beta_{i-1}, \beta_{i}\right],} & \text { при нечетных } i, \\
\left(\beta_{i-1}, \beta_{i}\right), & \text { при четных } i,\end{cases}
\end{gathered}
$$

В этом случае при выполнении условия (4) для четного $k$ получаем

$$
\begin{gathered}
P\left(x_{1}^{*}\right)=\left[\alpha_{0}, x_{p}\right] \cup\left[\beta_{0}, \beta_{1}\right] \cup\left[\alpha_{2}, \alpha_{1}\right] \cup \ldots \\
\ldots \cup\left[\alpha_{k}, \alpha_{k-1}\right] \cup\left[\beta_{k}, b\right] \cup\left[a, \alpha_{k+1}\right]
\end{gathered}
$$

для нечетного $k$ -

$$
\begin{gathered}
P\left(x_{1}^{*}\right)=\left[\alpha_{0}, x_{p}\right] \cup\left[\beta_{0}, \beta_{1}\right] \cup\left[\alpha_{2}, \alpha_{1}\right] \cup \ldots \\
\ldots \cup\left[\beta_{k-1}, \beta_{k}\right] \cup\left[\alpha_{k+1}, \alpha_{k}\right], \\
P\left(x_{2}^{*}\right)=[a, b] \backslash P\left(x_{1}^{*}\right) .
\end{gathered}
$$

В рассматриваемом случае притягивающих неподвижных точек $x_{1}^{*}$ и $x_{2}^{*}$ с $x_{1}^{*}<x_{p}<x_{2}^{*}$ при нарушении условия $f_{2} f_{1}(x)>x$ на $\left[a, \alpha_{0}\right]$ в уравнении (1) возможны циклы различных периодов. Существование циклов различных периодов связано с наличием неподвижных точек различных суперпозиций функций $f_{1}(x)$ и $f_{2}(x)$. Рассмотрим, например, условия существования циклов периода 2.

Пусть в (1) функции $f_{1}(x)$ и $f_{2}(x)$, убывающие соответственно на $\left[a, \alpha_{0}\right]$ и $\left[\beta_{0}, b\right]$, условие $f_{2} f_{1}(x)>x$ не выполняется на $\left[a, \alpha_{0}\right]$. В силу непрерывности и убывания $f_{1}(x)$ существует $\delta>0$ такое, что для любых $x \in\left(\alpha_{0}-\delta, \alpha_{0}\right)$ имеет место неравенство $x_{p}<f_{1}(x)<\beta_{0}$. Следовательно, на $\left(\alpha_{0}-\delta, \alpha_{0}\right)$ выполняются неравенства $f_{2} f_{1}(x)>x_{p}>\alpha_{0}>x$. Если отображение $f_{2} f_{1}$ имеет на $\left[a, \alpha_{0}\right]$ единственную неподвижную точку $z, f_{2} f_{1}(z)=z$, то в силу предыдущего $f_{2} f_{1}(x)>x$ на $\left[z, \alpha_{0}\right]$. Значит, $z$ не является притягивающей неподвижной точкой отображения $f_{2} f_{1}$. Если на $[a, z)$ выполняется неравенство $x<f_{2} f_{1}(x) \leq z$, то $z$ является полупритягивающей неподвижной точкой с левосторонней областью притяжения, равной $[a, z)$. Неподвижной точке $z$ отображения $f_{2} f_{1}$ соответствует цикл $\{z$, $\left.f_{1}(z)\right\}$ периода 2 уравнения (1), к которому при выполнении на $[a, z)$ условия $x<f_{2} f_{1}(x) \leq z$ сходятся решения уравнения (1), начинающиеся в $[a, z) \cup\left(f_{2}^{-1}(z), b\right]$. Если на $[a, z) f_{2} f_{1}(x)<x$, то к этому циклу ни одно из других решений уравнения (1) не сходится. Если отображение $f_{2} f_{1}$ на $\left[a, \alpha_{0}\right]$ имеет несколько неподвижных точек, то часть из них может обладать свойством притяжения или полупритяжения и соответствующие им циклы периода 2 уравнения (1) будут иметь непустые области притяжения. Следует отметить, что при фрункциях $f_{1}(x)$ и $f_{2}(x)$, убывающих соответственно на $\left[a, \alpha_{0}\right]$ и $\left[\beta_{0}, b\right]$, при невыполнении условия $f_{2} f_{1}(x)>x$ на $\left[a, \alpha_{0}\right]$ в уравнении (1) возможны только циклы периода 2. Существование у уравнения (1) циклов, например, периода 3 связано с наличием неподвижных точек отображения $f_{1}^{2} f_{2}$, при этом могут существовать и циклы периода 2.

Отсутствие неподвижных точек $\mathrm{c} f_{1}(x)>x$ на $\left[a, x_{p}\right]$ и $f_{2}(x)<x$ на $\left[x_{p}, b\right]$. В этом случае в силу непрерывности $f_{i}(x)$ и $f_{i}[a, b] \subset(a, b)$ функция $f_{1}(x)$ имеет неподвижные точки в $\left(x_{p}, b\right)$, а функция $f_{2}(x)-$ в $\left(a, x_{p}\right)$. Пусть множества $W_{0}, W, V_{0}, V$ и числа $\alpha_{0}, \beta_{0}$ имеют прежний смысл. Число $\alpha_{i}$ определяется как наибольшее на $\left[a, \alpha_{i-1}\right]$ решение уравнения $f_{i}(x)=\alpha_{i-1}$, а число $\beta_{i}$ - как наименьшее на $\left(\beta_{i-1}\right.$, b] решение уравнения $f_{2}(x)=\beta_{i-1}$, если таких решений нет, то, соответственно, принимаем $\alpha_{i}=a, \beta_{i}=b$. Строение промежутков $\left[a, x_{p}\right]$ и $\left(x_{p}, b\right]$ по переходам в $W_{0}$ и $V_{0}$ определяет следующее утверждение.

Лемма 4. Пусть в уравнении (1) фуункции $f_{i}(x)$ непрерывны на $[a, b], f_{i}[a, b] \subset(a, b)$, $f_{1}(x)>x$ на $\left[a, x_{p}\right]$ и $f_{2}(x)<x$ на $\left[x_{p}, b\right]$. Тогда

$$
\left[a, x_{p}\right]=\bigcup_{i=0}^{k+1} f_{1}^{-i} W_{0},
$$

где $k$ определяется неравенством $\alpha_{k}<\min _{\left[a, x_{p}\right]} f_{1}(x) \leq \alpha_{k-1}$ и каждое $f_{1}^{-(i-1)} W_{0}$ содержит промежуток, расположенный правее $f_{1}^{-i} W_{0}$;

$$
\left(x_{p}, b\right]=\bigcup_{i=0}^{m+1} f_{2}^{-i} V_{0},
$$

где $m$ определяется неравенством $\beta_{m-1}<\max _{\left[x_{p}, b\right]} f_{2}(x) \leq \beta_{m}$ и каждое $f_{2}^{-(i-1)} V_{0}$ содержит промежуток, расположенный левее $f_{2}^{-i} V_{0}$.

Из этого утверждения следует, что множества чисел $\alpha_{i}$ и $\beta_{i}$ конечны. Простейшую структуру промежутки $\left[\begin{array}{ll}a, & x_{p}\end{array}\right]$ $n\left(x_{p}, b\right]$ имеют для монотонных фрунк- 
ций $f_{i}(x)$. Например, если $f_{1}(x)$ возрастает на $\left[a, x_{p}\right]$ и $f_{1}(a)<x_{p}$, то $W_{0}=\left(\alpha_{0}, x_{p}\right]$, $f_{1}^{-i} W_{0}=\left(\alpha_{i}, \alpha_{i-1}\right], \quad i=1,2, \ldots, k+1, \alpha_{k+1}=$ a и $\left[a, x_{p}\right]=\left[a, \alpha_{k}\right] \cup\left(\alpha_{k}, \alpha_{k-1}\right] \cup \ldots \cup\left(\alpha_{0}, x_{p}\right]$. Если $f_{2}(x)$ возрастаетна $\left[x_{p}, b\right]$ и $f_{2}(b)>x_{p^{\prime}}$ то $V_{0}=\left(x_{p^{\prime}}\right.$ $\left.\beta_{0}\right], f_{2}^{-i} V_{0}=\left(\beta_{i-1}, \beta_{i}\right], i=1,2, \ldots, m+1, \beta_{m+1}=b$ и $\left(x_{p}, b\right]=\left(x_{p}, \beta_{0}\right] \cup\left(\beta_{0}, \beta_{1}\right] \cup \ldots \cup\left(\beta_{m}, b\right]$.

Знание структуры промежутков $\left[a, x_{p}\right]$ и $\left(x_{p}, b\right]$ позволяет определить условия наличия в (1) циклов различных периодов. Пусть $\eta_{i}$ наименьшее на $\left[x_{p}, b\right]$ решение уравнения $f_{2}(x)=\alpha_{i}$.

Теорема 2. Пусть в (1) фрункции $f_{1}(x)$ и $f_{2}(x)$ непрерывные и возрастающие соответственно на $\left[a, x_{p}\right]$ и $\left[x_{p}, b\right]$, на $\left[a, x_{p}\right] f_{1}(x)>x$, на $\left[x_{p}\right.$, b] $f_{2}(x)<x$. Тогда при выполнении неравенств

$$
f_{1}\left(x_{p}\right) \leq \beta_{0}, \quad f_{2}\left(x_{p}\right)>\alpha_{0}
$$

в (1) существуют только циклы $\left\{\gamma, f_{1}(\gamma)\right\}$ периода 2, где $\gamma$ - неподвижные точки отображения $f_{2} f_{1}$ на $\left(\alpha_{0}, x_{p}\right)$. Если при некотором і выполняются неравенства

$$
f_{1}\left(x_{p}\right) \leq \eta_{i}, \quad \alpha_{i+1} \leq f_{2}\left(x_{p}\right)<\alpha_{i}
$$

то в (1) существуют только циклы $\left\{\gamma, f_{1}(\gamma)\right.$, $\left.f_{2} f_{1}(\gamma), f_{1} f_{2} f_{1}(\gamma), \ldots, f_{1}^{i} f_{2} f_{1}(\gamma)\right\}$ периода $i+3$, где $\gamma$ - неподвижные точки отображения $f_{1}^{i+1} f_{2} f_{1}$ на $\left(\alpha_{0}, x_{p}\right]$. Если отображение $f_{2} f_{1}$ или $f_{1}^{i+1} f_{2} f_{1}$ имеет на $\left(\alpha_{0}, x_{p}\right)$ единственную неподвижную точку $\gamma$, то соответствующие циклы притягивающие и их областью притяжения является отрезок $[a, b]$.

Доказательство. Проведем его только для случая выполнения неравенств (9). При выполнении условий (8) доказательство проводится по аналогичной схеме. Пусть выполнено условие (9). В силу возрастания $f_{1}(x)$ и $f_{2}(x)$ соответственно на $\left[a, x_{p}\right]$ и $\left[x_{p}, b\right]$ их суперпозиция $f_{1}^{i+1} f_{2} f_{1}$, также возрастающая функция на $\left[\alpha_{0}, x_{p}\right]$. Рассмотрим отображение $f_{1}^{i+1} f_{2} f_{1}$ в точках $\alpha_{0}$ и $x_{p}$. По условиям утверждения $x_{p}<f_{1}\left(x_{p}\right) \leq \eta_{i}$. Из возрастания $f_{2}(x)$ имеем $f_{2} f_{1}\left(x_{p}\right) \leq f_{2}\left(\eta_{i}\right)=\alpha_{i}$, отсюдапо возрастанию $f_{1}(x)$ получаем цепочку неравенств $f_{1} f_{2} f_{1}\left(x_{p}\right) \leq f_{1}\left(\alpha_{i}\right)=\alpha_{i-1}, \ldots, f_{1}^{i+1} f_{2} f_{1}\left(x_{p}\right) \leq f_{1}\left(\alpha_{0}\right)=x_{p}$ ， т. e. $f_{1}^{i+1} f_{2} f_{1}\left(x_{p}\right) \leq x_{p}$. Из $\alpha_{i+1} \leq f_{2}\left(x_{p}\right)<\alpha_{i}$ в силу возрастания $f_{1}(x)$ и $f_{2}(x)$ получаем цепочку неравенств $\alpha_{i}=f_{1}\left(\alpha_{i+1}\right) \leq f_{1} f_{2}\left(x_{p}\right)$, $\alpha_{i-1}=f_{1}\left(\alpha_{i}\right) \leq f_{1}^{2} f_{2}\left(x_{p}\right), \ldots, \alpha_{0}=f_{1}\left(\alpha_{1}\right) \leq f_{1}^{i+1} f_{2}\left(x_{p}\right)$.

Отсюда с учетом $x_{p}=f_{1}\left(\alpha_{0}\right)$ имеет место неравенство $\alpha_{0} \leq f_{1}^{i+1} f_{2} f_{1}\left(\alpha_{0}\right)$. В силу леммы 4 решения из $\left[a, x_{p}\right]$ или начинаются в $W_{0}=\left(\alpha_{0}\right.$, $\left.x_{p}\right]$, или попадают в $W_{0}$ за конечное число шагов. Для любых $x \in\left(\alpha_{0}, x_{p}\right]$ в силу возрастания $f_{1}^{i+1} f_{2} f_{1}$ имеет место неравенство $\alpha_{0}<f_{1}^{i+1} f_{2} f_{1}(x) \leq x_{p}$. Из непрерывности и возрастания отображения $f_{1}^{i+1} f_{2} f_{1}$ на $\left[\alpha_{0}, x_{p}\right]$ и неравенств $\alpha_{0} \leq f_{1}^{i+1} f_{2} f_{1}\left(\alpha_{0}\right), f_{1}^{i+1} f_{2} f_{1}\left(x_{p}\right) \leq x_{p}$ следует, что при выполнении условий (9) это отображение имеет на $\left(\alpha_{0}, x_{p}\right]$ неподвижные точки $\gamma$. Следовательно, в уравнении (1) существуют циклы $\left\{\gamma, f_{1}(\gamma), f_{2} f_{1}(\gamma), f_{1} f_{2} f_{1}(\gamma), \ldots, f_{1}^{i} f_{2} f_{1}(\gamma)\right\}$ периода $i+3$. Если отображение $f_{1}^{i+1} f_{2} f_{1}$ имеет единственную неподвижную точку, то в силу перечисленных его свойств этот цикл является притягивающим с областью притяжения, равной отрезку $[a, b]$.

Следствие 1. Пусть для уравнения (1) выполнены условия теоремы 2, $f_{1}(x)$ и $f_{2}(x)$ выпуклые (вогнутые) функции соответственно на $\left[a, x_{p}\right]$ и $\left[x_{p}, b\right]$. Тогда при выполнении неравенств (8) уравнение (1) имеет единственный цикл $\left\{\gamma, f_{1}(\gamma)\right\}$, который является притягивающим на всем отрезке [a, b]. При выполнении неравенств (9) уравнение (1) имеет единственный цикл $\left\{\gamma, f_{1}(\gamma), f_{2} f_{1}(\gamma), f_{1} f_{2} f_{1}(\gamma), \ldots, f_{1}^{i} f_{2} f_{1}(\gamma)\right\}$, который является притягивающим на всем отрезке [a, b].

Теорема 3. Пусть в уравнении (1) функции $f_{1}(x)$ и $f_{2}(x)$ непрерывные и убывающие соответственно на $\left[a, x_{p}\right] и\left[x_{p}, b\right], f_{1}\left(x_{p}\right)>x_{p}, f_{2}\left(x_{p}\right)<$ $<x_{p^{\prime}} f_{1}(a)<b$ и $f_{2}(b)>a$, тогда это уравнение имеет только циклы $\left\{\gamma, f_{1}(\gamma)\right\}$ периода 2, где $\gamma$ - неподвижная точка отображения $f_{2} f_{1}$ на $\left[a, x_{p}\right]$. Если при этом фрункция $f_{1}(x)$ выпуклая (вогнутая) на $\left[a, x_{p}\right]$, a $f_{2}(x)$ - вогнутая (выпуклая) на $\left[x_{p}, b\right]$, то такой цикл единственный и его областью притяжения является отрезок $[a, b]$.

\section{СПИСОК ИСПОЛЬЗОВАННОЙ ЛИТЕРАТУРЫ}

1. Шарковский А. Н. Разностные уравнения и их приложения / А. Н. Шарковский, Ю. Л. Майстренко, Е. Ю. Романенко. - Киев : Наукова думка, 1986. - 280 с.

2. Шапиро А. П. Рекуррентные уравнения в популяционной биологии / А. П. Шапиро, С. П. Луппов. - М. : Наука, 1983. - 133 с.

3. Свирежев Ю. М. Устойчивость биологических сообществ / Ю. М. Свирежев, Д. О. Логофет. - М. : Наука, 1978. - 352 с.

4. Якобсон М. В. О свойствах динамических систем, порождаемых отображениями вида $x \rightarrow A x e^{-x} /$ М. В. Якобсон // Моделирование биологических сообществ : сб. ст. / отв. ред. А. П. Шапиро. - Владивосток : Изд-во Дальневост. науч. центра АН СССР, 1975. - С. 141-162. 
5. Li T.-J. Period three implies chaos / T.-J. Li, J. A. Yorke // American Mathematical Monthly. — 1975. Vol. 82, № 10. - P. 982-985.

6. Абдуллин Р. З. Области притяжения стационаров разностного уравнения с переключениями / Р. 3. Абдуллин / / Автоматика и телемеханика. - 2008. - № 3. - С. 126-134.

\section{REFERENCES}

1. Sharkovskii A. N., Maistrenko Yu. L., Romanenko E. Yu. Raznostnye uravneniya i ikh prilozheniya [Difference Equations and Their Applications]. Kiev, Naukova dumka Publ., 1986. 280 p.

2. Shapiro A. P., Luppov S. P. Rekurrentnye uravneniya v populyatsionnoi biologii [Recurrence Equations in Population Biology]. Moscow, Nauka Publ., 1983. 133 p.

3. Svirezhev Yu. M., Logofet D. O. Ustoichivost' biologicheskikh soobshchestv [Stability of Biological Communities]. Moscow, Nauka Publ., 1978. 352 p.

4. Yakobson M. V. On Properties of Dynamical Systems Generated by the Mapping $x \rightarrow A x e^{-x}$. In Shapiro A. P. (ed.). Modelirovanie biologicheskikh soobshchestv [Modelling of Biological Communities]. Vladivostok, The Far-Eastern Scientific Centre of the Academy of Sciences of the USSR Publ., 1975, pp. 141-162. (In Russian).

5. Li T.-J., Yorke J. A. Period three implies chaos. American Mathematical Monthly, 1975, vol. 82, no. 10, pp. 982-985.

6. Abdullin R. Z. Attraction domains of the stationary points of the difference equation with switchings. Avtomatika i telemekhanika = Automation and Remote Control, 2008, no. 3, pp. 126-134. (In Russian).

\section{Информация об авторе}

Абдуллин Рафраэль Зинатович - кандидат физико-математических наук, доцент, кафедра математики и эконометрики, Байкальский государственный университет, 664003, г. Иркутск, ул. Ленина, 11, e-mail: abdullin-rz@bgu.ru.

\section{Для цитирования}

Абдуллин Р. З. Стационары и циклы разностного уравнения с разрывной правой частью / Р. З. Абдуллин / / Известия Байкальского государственного университета. - 2017. - Т. 27, № 4. - С. 594-599. DOI: $10.17150 / 2500-2759.2017 .27(4) .594-599$.

\section{Author}

Rafael Z. Abdullin - PhD in Physics and Mathematics, Associate Professor, Baikal State University, 11 Lenin St., 664003, Irkutsk, Russian Federation, e-mail: abdullin-rz@bgu.ru.

\section{For citation}

Abdullin R. Z. Steady States and Cycles of a Difference Equation with Discontinuous Righthand Side. Izvestiya Baykal'skogo gosudarstvennogo universiteta $=$ Bulletin of Baikal State University, 2017, vol. 27, no. 4, pp. 594-599. DOl: 10.17150/25002759.2017.27(4).594-599. (In Russian). 